\title{
PERIODIC PARABOLIC PROBLEMS WITH NONLINEARITIES INDEFINITE IN SIGN
}

\author{
T. Godoy and U. Kaufmann
}

Abstract

Let $\Omega \subset \mathbb{R}^{N}$ be a smooth bounded domain. We give sufficient conditions (which are also necessary in many cases) on two nonnegative functions $a, b$ that are possibly discontinuous and unbounded for the existence of nonnegative solutions for semilinear Dirichlet periodic parabolic problems of the form $L u=$ $\lambda a(x, t) u^{p}-b(x, t) u^{q}$ in $\Omega \times \mathbb{R}$, where $0<p, q<1$ and $\lambda>0$. In some cases we also show the existence of solutions $u_{\lambda}$ in the interior of the positive cone and that $u_{\lambda}$ can be chosen such that $\lambda \rightarrow u_{\lambda}$ is differentiable and increasing. A uniqueness theorem is also given in the case $p \leq q$. All results remain valid for the corresponding elliptic problems.

\section{Introduction}

Let $\Omega$ be a $C^{2+\theta}$ bounded domain in $\mathbb{R}^{N}, N \geq 2, \theta \in(0,1)$. For $T>0$ and $1 \leq p \leq \infty$, let $L_{T}^{p}$ be the Banach space of $T$-periodic functions $f$ on $\Omega \times \mathbb{R}$ (i.e. satisfying $f(x, t)=f(x, t+T)$ a.e. $(x, t) \in \Omega \times \mathbb{R}$ ) such that $f_{\mid \Omega \times(0, T)} \in L^{p}(\Omega \times(0, T))$, equipped with the norm $\|f\|_{L_{T}^{p}}:=$ $\left\|f_{\mid \Omega \times(0, T)}\right\|_{L^{p}(\Omega \times(0, T))}$. Let $C_{T}$ be the space of continuous and $T$-periodic functions on $\bar{\Omega} \times \mathbb{R}$ provided with the $L^{\infty}$ norm, and let $C_{T}^{1+\theta,(1+\theta) / 2}$ be the space of $T$-periodic functions belonging to $C^{1+\theta,(1+\theta) / 2}(\bar{\Omega} \times \mathbb{R})$.

Let $\left\{a_{i j}\right\},\left\{b_{j}\right\}, 1 \leq i, j \leq N$, be two families of $T$-periodic functions satisfying $a_{i j} \in C^{0,1}(\bar{\Omega} \times \mathbb{R}), a_{i j}=a_{j i}$ and $b_{j} \in L_{T}^{\infty}$, and assume that

$$
\sum a_{i j}(x, t) \xi_{i} \xi_{j} \geq \alpha_{0}|\xi|^{2}
$$

for some $\alpha_{0}>0$ and all $(x, t) \in \Omega \times \mathbb{R}, \xi \in \mathbb{R}^{N}$. Let $A$ be the $N \times N$ matrix whose $i, j$ entry is $a_{i j}$, let $\bar{b}=\left(b_{1}, \ldots, b_{N}\right)$, let $0 \leq c_{0} \in L_{T}^{r}, r>N+2$,

2000 Mathematics Subject Classification. 35K20, 35P05, 35B10.

Key words. Periodic, parabolic, indefinite.

Partially supported by CONICET, SECYT-UNC, ANPCYT and Fundación Antorchas. 
and let $L$ be the parabolic operator given by

$$
L u=u_{t}-\operatorname{div}(A \nabla u)+\langle\bar{b}, \nabla u\rangle+c_{0} u .
$$

Let $W=\left\{u \in L^{2}\left((0, T), H_{0}^{1}(\Omega)\right): u_{t} \in L^{2}\left((0, T), H^{-1}(\Omega)\right)\right\}$. For a given $f \in L_{T}^{2}$, we will say that $u$ is a (weak) solution of the periodic problem

$$
\begin{cases}L u=f & \text { in } \Omega \times \mathbb{R} \\ u=0 & \text { on } \partial \Omega \times \mathbb{R} \\ u T \text {-periodic } & \end{cases}
$$

if $u$ is $T$-periodic, $u_{\mid \Omega \times(0, T)} \in W$ and

$$
\int_{\Omega \times(0, T)}\left[-u \frac{\partial h}{\partial t}+\langle A \nabla u, \nabla h\rangle+\langle\bar{b}, \nabla u\rangle h+c_{0} u h\right]=\int_{\Omega \times(0, T)} f h
$$

for all $h \in C_{c}^{\infty}(\Omega \times(0, T))$. For $u \in W$, the inequality $L u \geq f$ (resp. $\leq$ ) in $\Omega \times \mathbb{R}, u=0$ in $\partial \Omega \times \mathbb{R}$ will be understood in the analogous weak sense.

For $1 \leq r \leq \infty$ let $W_{r}^{2,1}\left(\Omega \times\left(t_{0}, t_{1}\right)\right)$ be the Sobolev space of the functions $u \in L^{r}\left(\Omega \times\left(t_{0}, t_{1}\right)\right), u=u(x, t), x=\left(x_{1}, \ldots, x_{N}\right)$ such that $u_{t}, u_{x_{j}}$ and $u_{x_{i} x_{j}}$ belong to $L^{r}\left(\Omega \times\left(t_{0}, t_{1}\right)\right)$ for $1 \leq i, j \leq N$, and let $W_{r, T}^{2,1}$ be the space of $T$-periodic functions such that $u_{\mid \Omega \times(0, T)} \in$ $W_{r}^{2,1}(\Omega \times(0, T))$. For $f \in L_{T}^{r}, r>1$, we say that $u$ is a strong solution of $(1.1)$ if $u \in W_{r, T}^{2,1}(\Omega \times \mathbb{R})$ and the equation holds a.e. in the pointwise sense.

The existence of positive solutions for periodic parabolic problems of the form

$$
\begin{cases}L u=g(x, t, u) & \text { in } \Omega \times \mathbb{R} \\ u=0 & \text { on } \partial \Omega \times \mathbb{R} \\ u T \text {-periodic } & \end{cases}
$$

has been widely studied (see e.g. [15] and the references therein). For applications we refer to $[\mathbf{1 5}]$, [5]. In [11] and [13], bifurcation of positive solutions for $(1.2)$ was proved assuming that $\xi \rightarrow g(x, t, \xi) / \xi$ is nonincreasing in $(0, \infty)$ and that $g_{\xi}(x, t, 0)$ belongs to $L_{T}^{r}$ for some $r>(N+2) / 2$. On the other hand, in [12], existence results of positive solutions for (1.2) were given without monotonicity conditions on $g$ and allowing $g_{\xi}(x, t, 0)=+\infty$, but assuming that $\inf _{0<\sigma \leq \xi}(g(x, t, \sigma) / \sigma)$ belongs to $L_{T}^{r}$ for some $r>(N+2) / 2$. However, in many cases of interest 
neither of the above conditions hold. A typical example of this situation is the problem

$$
\begin{cases}L u=\lambda a(x, t) u^{p}-b(x, t) u^{q} & \text { in } \Omega \times \mathbb{R} \\ u=0 & \text { on } \partial \Omega \times \mathbb{R} \\ u T \text {-periodic } & \end{cases}
$$

where $a, b$ are two nonnegative functions, $0<p, q<1$ and $\lambda>0$.

Our aim in this paper is to study (1.3), where $a, b$ belong to $L_{T}^{r}$ with $r>N+2$. Concerning this problem, we will show existence of nonnegative solutions for all $\lambda>0$ under weak conditions on $a$ and $b$ (see Theorem 3.2 and also Remark 3.3), using iterative and fixed point methods combined with some facts about linear problems with weight. Also, under different assumptions on $a$ and $b$, we will prove existence of solutions in the interior of the positive cone of $C_{T}^{1+\theta,(1+\theta) / 2}$ for $\lambda$ large enough and, for $p \leq q$, that $u$ can be chosen such that $\lambda \rightarrow u_{\lambda}$ is differentiable and increasing (see Theorem 3.4). These last results will follow from a sub and supersolution approach together with the implicit function theorem. Finally, a uniqueness theorem for the solutions in the interior of the positive cone is given in Theorem 3.5 for the case $p \leq q$.

To avoid unnecessary complexity, we restrict ourselves to (1.3), but one can see that most of the results are still valid for increasing nonlinearities that behave like $u^{p}$ near the origin and infinity. We mention also that as a consequence of our proofs all results remain true for the analogous elliptic problem.

In order to relate our results to others in the literature, let us mention that similar elliptic problems have been studied for example in [20] for $L=-\Delta$ and $a, b \in C(\bar{\Omega})$ using a variational approach, and recently for $a, b \in C^{1}(\Omega)$ and allowing these functions to have a singularity in the boundary in [14, Section 3] (see also the references therein). The particular case $L u=m u^{p}$ in $\Omega$, with $0<p<1$ and $m$ changing sign, was treated in detail in $[\mathbf{2}]$ for $L=-\Delta$ and $m \in C^{\theta}(\bar{\Omega}), \theta \in(0,1)$, using sub and supersolutions to construct nonnegative solutions, and there is also a result for the associated parabolic initial boundary value problem there. For the one dimensional elliptic problem (1.3), a precise description of the solution set when $p>q$ and $a=b \equiv 1$ was given in [8] for the operator $L u=-\left(\left|u^{\prime}\right|^{s-2} u^{\prime}\right)^{\prime}, s>1$. On the other hand, in the periodic parabolic case, a similar problem to (1.3) with $p=q$ was considered extensively in [16] for the heat operator with Neumann boundary condition, and it is also shown there that the nonnegative solutions are 
not necessarily unique. Existence and multiplicity of changing sign solutions for similar periodic parabolic problems were also considered in [17]. We finally mention that related elliptic superlinear problems have been widely studied too, see e.g. [1] and references therein.

\section{Preliminaries and auxiliary results}

We start collecting some known facts about the (weak) solution operator (denoted by $L^{-1}$ ) and strong solutions for problem (1.1).

Remark 2.1. (i) For $r>(N+2) / 2$ it holds that $L^{-1}\left(L_{T}^{r}\right) \subset C_{T}$ and $L^{-1}: L_{T}^{r} \rightarrow C_{T}$ is a compact and positive operator (see e.g. [4, Section 5], also [11, Remarks 2.1 and 2.2]).

(ii) Given $f \in L_{T}^{r}, r>N+2$, there exists a unique solution $u \in W_{r, T}^{2,1}$ of (1.1) and the operator $L^{-1}: L_{T}^{r} \rightarrow W_{r, T}^{2,1}$ is continuous (see e.g. $[\mathbf{1 9}$, Section 4]). Moreover, from the Sobolev imbedding theorems (e.g. [18, Lemma 3.3, p. 80]) it follows that $u \in C_{T}^{1+\theta,(1+\theta) / 2}$; and if $f \geq 0$, the strong maximum principle (e.g. [5, Theorem 13.5]) gives that $u>0$ in $\Omega \times \mathbb{R}$ and $\frac{\partial u}{\partial \nu}<0$ on $\partial \Omega \times \mathbb{R}$, where $\nu$ denotes the outward unit normal to $\partial \Omega$.

We recall also some necessary facts about periodic parabolic problems with weight.

Remark 2.2. (i) Let $m \in L_{T}^{r}, r>(N+2) / 2$, and let

$$
P(m):=\int_{0}^{T} \operatorname{esssup}_{x \in \Omega} m(x, t) d t .
$$

Then $P(m)>0$ is necessary and sufficient for the existence of a (unique) positive principal eigenvalue $\lambda_{1}(m)$ for the periodic problem $L u=\lambda m u$ in $\Omega \times \mathbb{R}, u=0$ on $\partial \Omega \times \mathbb{R}($ cf. [10, Theorem 3.6]).

(ii) For $\lambda \in \mathbb{R}$, let $\mu_{m}(\lambda)$ be defined as the unique $\mu \in \mathbb{R}$ such that the Dirichlet periodic problem $L u=\lambda m u+\mu_{m}(\lambda) u$ in $\Omega \times \mathbb{R}$ has a positive solution $u$. Then $\mu_{m}(\lambda)$ is well defined, $\mu_{m}(0)>0$, $\mu_{m}$ is concave and continuous, and a given $\lambda \in \mathbb{R}$ is a principal eigenvalue associated to the weight $m$ if and only if $\mu_{m}(\lambda)=0$ (cf. [10, Lemma 3.2]). Also, if $\lambda_{1}(m)$ exists, then for $\lambda>0$, $\mu_{m}(\lambda)>0$ if and only if $\lambda<\lambda_{1}(m)$, and if $\lambda_{1}(m)$ does not exist, $\mu_{m}(\lambda)>0$ for all $\lambda>0$. 
(iii) If $\mu_{m}(\lambda)>0$ then for all $h \in L_{T}^{r}$ the $T$-periodic problem

$$
\begin{cases}L u=\lambda m u+h & \text { in } \Omega \times \mathbb{R} \\ u=0 & \text { on } \partial \Omega \times \mathbb{R} \\ u T \text {-periodic } & \end{cases}
$$

has a unique solution $u \in W_{r, T}^{2,1}$, which is positive if $h>0$, and the solution operator $h \rightarrow u$ for this problem is continuous from $L_{T}^{r}$ into $C_{T}$ (cf. [11, Lemma 2.9]). In particular, these conclusions apply for all $\lambda \geq 0$ if $\lambda_{1}(m)$ does not exist and for $0 \leq \lambda<\lambda_{1}(m)$ when $\lambda_{1}(m)$ exists.

Conversely, if (2.1) has a positive solution $h$ and $\lambda_{1}(m)$ exists, then $\lambda<\lambda_{1}(m)$ (cf. [13, Remark 2.1 (e)]).

(iv) The following comparison principle holds: if $m_{1}, m_{2} \in L_{T}^{r}, P\left(m_{1}\right)>$ 0 and $m_{1} \leq m_{2}$ in $\Omega \times \mathbb{R}$, then $\lambda_{1}\left(m_{1}\right) \geq \lambda_{1}\left(m_{2}\right)$ and, if in addition $m_{1}<m_{2}$ in a set of positive measure, then $\lambda_{1}\left(m_{1}\right)>\lambda_{1}\left(m_{2}\right)$ (cf. [10, Remark 3.7]).

The following lemma states a maximum principle for noncylindrical domains.

Lemma 2.3. Let $D \subset \Omega \times \mathbb{R}$ be a domain, let $0 \leq g \in L^{r}(D)$, $r>N+2$, and let $u \in W_{r, T}^{2,1}(\Omega \times \mathbb{R})$ such that

Then $u \geq 0$ in $D$.

$$
\begin{cases}L u=g & \text { in } D \\ u \geq 0 & \text { on } \partial D\end{cases}
$$

Proof: Since $u \in C(\bar{D})$, there exists $c:=\min \bar{D} u$. Suppose $c<0$, and let $\left(x_{0}, t_{0}\right) \in \bar{D}$ such that $u\left(x_{0}, t_{0}\right)=c$. Then $\left(x_{0}, t_{0}\right) \in D$. Let $Q \subset D$ be a smooth bounded cylinder of the form $Q=\Omega_{0} \times\left(t_{0}-\delta, t_{0}+\delta\right)$, $\delta>0$, such that $x_{0} \in \partial \Omega_{0}$. Taking into account that $\nabla u\left(x_{0}, t_{0}\right)=$ 0 , the maximum principle in Proposition 13.2 in [5] says that $u \equiv c$ in $Q$. Now, choosing adequately such $Q$ 's (for example, taking $\partial \Omega_{0}$ "planar" around $\left.x_{0}\right)$ we can cover a neighborhood $B_{\rho}\left(x_{0}\right) \times\left(t_{0}-\delta, t_{0}+\delta\right)$ with a finite number of them, and hence it follows that the set $C:=$ $\{(x, t) \in D: u(x, t)=c\}$ is open. Since it is also closed we have $C=D$. But then $u<0$ on $\partial D$. Contradiction.

Remark 2.4. In Lemma 2.3, the regularity assumptions on the coefficients of $L$ can be weakened. In fact, it is clear from the proof that it suffices that they hold in each compact subset of $D$. 


\section{The main results}

Lemma 3.1. Let $b, f \in L_{T}^{r}, r>N+2, b \geq 0$. Then, there exists a unique $u \in W_{r, T}^{2,1}$ solution of

$$
\begin{cases}L u+b|u|^{q-1} u=f & \text { in } \Omega \times \mathbb{R} \\ u=0 & \text { on } \partial \Omega \times \mathbb{R} \\ u T \text {-periodic. } & \end{cases}
$$

Moreover, the solution operator $S: C_{T} \rightarrow C_{T}$ is compact and increasing. In particular, $f>0$ implies $S f>0$.

Proof: Let $v \in C_{T}$. From Remark 2.1 (ii) we have that there exists a unique solution $u \in W_{r, T}^{2,1}$ of the Dirichlet periodic problem

$$
L u+b|v|^{q-1} v=f \quad \text { in } \Omega \times \mathbb{R} .
$$

We note that the solution operator $S_{f}: C_{T} \rightarrow C_{T}$ is compact. Indeed, Lebesgue's dominated convergence theorem gives that the map $v \rightarrow$ $|v|^{q-1} v$ is continuous from $C_{T}$ into $L_{T}^{s}$ for all $s \geq 1$, and therefore $v \rightarrow-b|v|^{q-1} v+f$ is continuous from $C_{T}$ into $L_{T}^{l}$ for $l>N+2$. Thus, the compactness follows from Remark $2.1 \mathrm{i}$ ).

For $R>0$, let $\bar{B}_{R}^{C_{T}}$ be the closed ball in $C_{T}$ with center at 0 and radius $R$. We claim that $S_{f}: \bar{B}_{R}^{C_{T}} \rightarrow \bar{B}_{R}^{C_{T}}$ for $R$ large enough. Indeed, from (3.2) we have $|L u| \leq|f|+R^{q} b$ and thus

$$
-\left(L^{-1}|f|+R^{q} L^{-1} b\right) \leq u \leq L^{-1}|f|+R^{q} L^{-1} b .
$$

Hence, $|u| \leq c_{1}+c_{2} R^{q}$ where $c_{1}, c_{2}$ do not depend on $u$ and the claim follows. So, the Schauder fixed point theorem (e.g. [9, Corollary 11.2]) gives a solution for (3.1).

Suppose now there exist two solutions $u, w \in W_{r, T}^{2,1}$ for (3.1). Then

$$
L(u-w)=b\left(|w|^{q-1} w-|u|^{q-1} u\right) \quad \text { in } \Omega \times \mathbb{R} .
$$

Since $\left(|w|^{q-1} w-|u|^{q-1} u\right) /(w-u)$ is always positive when $u \neq w$, the maximum principle in Lemma 2.3 implies $u=w$.

To end the lemma, we note that the compactness of $S$ follows reasoning as in the beginning of the proof, and the fact that $S$ is increasing follows by Lemma 2.3 in a similar way as above. 
Theorem 3.2. Let $0<p, q<1$, and let $0 \leq a, b \in L_{T}^{r}, r>N+2$, such that the following condition holds

(H1) There exists an open set $B:=\Omega_{0} \times\left(t_{0}, t_{1}\right) \subset \Omega \times \mathbb{R}$ such that $a(x, t)>0$ in a subset of $B$ of positive measure and $b \equiv 0$ in $B$.

Then, for all $\lambda>0$ there exists a (nontrivial) nonnegative solution $u \in W_{r, T}^{2,1}$ of (1.3).

Proof: Let $0 \leq v \in C_{T}$ and $\lambda>0$. By the above lemma there exists a unique positive solution $u \in W_{r, T}^{2,1}$ of (3.1) with $f:=\lambda a v^{p}$. Let $S_{f}$ be the operator defined by $S_{f} v=S\left(\lambda a v^{p}\right)$ where $S$ is the solution operator of Lemma 3.1. Then $S_{f}$ is compact and nondecreasing. Note that if $v \equiv c$ for some constant $c$ large enough, then $S_{f} v \leq v$. Indeed, let $u=S_{f} v$. From (3.1) it follows that $L u \leq \lambda a v^{p}$ and so $\|u\|_{\infty} \leq K c^{p}$ for some $K$ independent of $c$.

Take $v \equiv c \gg 0$, consider the nonincreasing sequence $\left\{S_{f}^{j} v\right\}_{j=0}^{\infty}$ and let $u_{\infty} \geq 0$ be its limit. We have

$$
\begin{cases}L u_{j+1}=\lambda a u_{j}^{p}-b u_{j+1}^{q} & \text { in } \Omega \times \mathbb{R} \\ u_{j+1}=0 & \text { on } \partial \Omega \times \mathbb{R} \\ u_{j+1} T \text {-periodic, } & \end{cases}
$$

where $u_{j}=S_{f}^{j} v$. Now, by Lebesgue's theorem the right side of (3.3) converges in $L_{T}^{r}$, thus $\left\{u_{j+1}\right\}_{j=1}^{\infty}$ converges in $W_{r, T}^{2,1}$ (and so also in $C_{T}$ ). Then $u_{\infty} \in W_{r, T}^{2,1}$ and $u_{\infty}=0$ on $\partial \Omega \times \mathbb{R}$. Going to the limit in (3.3) we find that $L u_{\infty}=\lambda a u_{\infty}^{p}-b u_{\infty}^{q}$ in $\Omega \times \mathbb{R}$. It remains to see that $u_{\infty} \neq 0$. We proceed by contradiction. Suppose that $u_{\infty}=0$. Since $u_{j}$ converges to $u_{\infty}$ in $C_{T}$, there exists $j_{0}$ such that $u_{j} \leq 1$ for $j \geq j_{0}$. Observe also that

$$
u_{k}(x, t)>0 \text { for }(x, t) \in B, \quad k \in \mathbb{N} \cup\{0\} .
$$

Indeed, (3.4) clearly holds for $k=0$ and if it holds for $k=j$, since

$$
\begin{cases}L u_{j+1}=\lambda a u_{j}^{p}>0 & \text { in } B \\ u_{j+1} \geq 0 & \text { on } \partial_{P} B \\ u_{j+1} T \text {-periodic, } & \end{cases}
$$

where

$$
\partial_{P} B:=\left(\partial \Omega_{0} \times\left(t_{0}, t_{1}\right)\right) \cup\left(\Omega_{0} \times\left\{t_{0}\right\}\right),
$$


the maximum principle gives (3.4) for $k=j+1$. Taking into account (3.4) and that $u_{j+1} \leq u_{j}$, a computation gives, for $p<s<1$ and $j \geq j_{0}$,

$$
\begin{aligned}
L\left(u_{j+1}^{1-s}\right)= & (1-s) u_{j+1}^{-s} L\left(u_{j+1}\right) \\
& +s(1-s) u_{j+1}^{-s-1}\left\langle A \nabla u_{j+1}, \nabla u_{j+1}\right\rangle+s c_{0} u_{j+1}^{1-s} \\
\geq & (1-s) u_{j+1}^{-s} \lambda a u_{j}^{p} \\
\geq & (1-s) \lambda a u_{j}^{p-s} \\
\geq & (1-s) \lambda a
\end{aligned}
$$

Also,

$$
u_{j+1} \geq 0 \text { on } \partial_{P} B
$$

From (3.6) and (3.7), the maximum principle implies $u_{j+1}^{1-s} \geq z$ on $B$ for $j \geq j_{0}$ where $z \in W_{r}^{2,1}(B)$ is the (nonnegative and nontrivial) solution of

$$
\begin{array}{ll}
L z=(1-s) \lambda a & \text { in } B, \\
z=0 & \text { on } \partial_{P} B .
\end{array}
$$

Thus $u_{\infty}^{1-s} \geq z$ and so $u_{\infty}=0$ is impossible.

Remark 3.3. Let us make some comments on the condition (H1). Let $m \in L_{T}^{r}, r>N+2, m=m^{+}-m^{-}$, where as usual $m^{+}=\max (m, 0)$ and $m^{-}=\max (-m, 0)$. By the above theorem there exists a nonnegative solution of the Dirichlet periodic problem

$$
L u=\lambda m^{+} u^{p}-m^{-} u^{q} \quad \text { in } \Omega \times \mathbb{R}
$$

for all $\lambda>0$ provided that (H1) holds. Moreover, if $m \in C_{T}$, (H1) is also necessary by the maximum principle. In particular, taking $\lambda=1$ and $p=q$ it follows that $L u=m u^{p}$ in $\Omega \times \mathbb{R}$ has a nonnegative solution if and only if $m>0$ at some point. This generalizes (and gives a different proof of) the existence results known in the elliptic case (e.g. [2]) as well as in the periodic parabolic case.

We focus now on the existence of strictly positive solutions for (1.3). We note that, for general $p, q \in(0,1)$, the only information available from the proof of Theorem 3.2 about the solution $u$ provided there is that $u(x, t)>0$ for each $B$ satisfying (H1) which, in general, does not implies that $u(x, t)>0$ for all $(x, t) \in \Omega \times \mathbb{R}$. 
Let

$$
\begin{aligned}
\Omega_{\varepsilon} & :=\{x \in \Omega: \delta(x)>\varepsilon\}, \\
A_{\varepsilon} & :=\Omega-\Omega_{\varepsilon} . \\
\delta(x) & :=\operatorname{dist}(x, \partial \Omega) \\
P^{\circ} & :=\text { interior of the positive cone of } C_{T}^{1+\theta,(1+\theta) / 2} .
\end{aligned}
$$

Theorem 3.4. Let $0<p, q<1$, and let $0 \leq a, b \in L_{T}^{r}, r>N+2$. Assume

(H2) Either there exist $\varepsilon, K>0$ and $\gamma>1-q-1 /(N+2)$ such that $b \leq K \delta^{\gamma}$ a.e. $(x, t) \in A_{\varepsilon} \times \mathbb{R}$, or $q>1+1 / r-1 /(N+2)$.

Then, there exists $\lambda_{0}>0$ such that for all $\lambda>\lambda_{0}$ problem (1.3) has a solution $u \in W_{r, T}^{2,1} \cap P^{\circ}$.

Assume in addition that

(H3) $p \leq q$, and either there exist $\varepsilon, K>0$ and $\gamma>1-p-1 /(N+2)$ such that $a \leq K \delta^{\gamma}$ a.e. $(x, t) \in A_{\varepsilon} \times \mathbb{R}$, or $p>1+1 / r-1 /(N+2)$.

Then, there exists $\lambda_{0}>0$ such that a solution $u=u_{\lambda}$ of $(1.3)$ can be chosen such that $\lambda \rightarrow u_{\lambda}$ is a $C^{1}$ increasing map from $\left(\lambda_{0}, \infty\right)$ into $W_{r, T}^{2,1} \cap P^{\circ}$.

Proof: Assume in (H1) that $e, K$ and $\gamma$ exist and let $v \in C_{T}$ such that $v \geq c \delta$ for some $c>0$. Since $1-q<\gamma+1 /(N+2)$ we can pick $\alpha$ and $\sigma$ with $\sigma>N+2$ such that $1-q<\alpha<\gamma+1 / \sigma$. Now,

$\left\|b / v^{\alpha}\right\|_{L^{\sigma}\left(A_{\varepsilon} \times(0, T)\right)}^{\sigma}=\int_{A_{\varepsilon} \times(0, T)} b^{\sigma} / v^{\alpha \sigma} \leq c^{-\alpha \sigma} K^{\sigma} \int_{A_{\varepsilon} \times(0, T)} \delta^{\sigma(\gamma-\alpha)}<\infty$ the last inequality because $\sigma(\gamma-\alpha)>-1$. So, $b / v^{\alpha} \in L_{T}^{s}$ for $s:=$ $\min (\sigma, r)>N+2$. If in (H1) it holds that $q>1+1 / r-1 /(N+2)$ then $1-q<1 / s-1 / r$ for some $s>N+2$ and so we can pick $\alpha$ such that $1-q<\alpha<1 / s-1 / r$. Now, by Hölder's inequality,

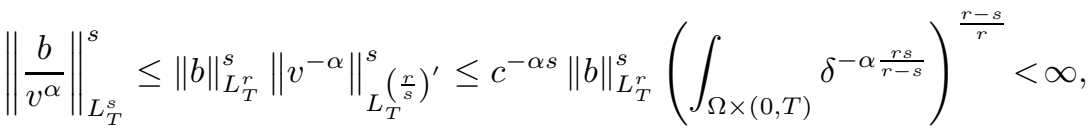
the last inequality because $\alpha \frac{r s}{r-s}<1$. Thus in any case we have $b / v^{\alpha} \in$ $L_{T}^{s}$ for some $\alpha>1-q$ and $s>N+2$. Then Theorem 3.4 in [12] gives a positive solution $u_{\lambda} \in L_{T}^{\infty}$ for the problem

$$
\begin{cases}\left(L+b / v^{\alpha}\right) u_{\lambda}=\lambda a u_{\lambda}^{p} & \text { in } \Omega \times \mathbb{R} \\ u_{\lambda}=0 & \text { on } \partial \Omega \times \mathbb{R} \\ u_{\lambda} T \text {-periodic, } & \end{cases}
$$


for all $\lambda>0$. Recalling Remark 2.1 (ii) we have that $u_{\lambda} \in P^{\circ}$, and, due to the homogeneity, it holds that $u_{\lambda}=\lambda^{1 /(1-p)} U$ for some $U \in P^{\circ}$ solution of $\left(L+b / v^{\alpha}\right) U=a U^{p}$ in $\Omega \times \mathbb{R}, U=0$ on $\partial \Omega \times \mathbb{R}$. Hence, since $\alpha \geq 1-q$, there exists $\lambda_{0}>0$ such that $u_{\lambda} \geq v^{\alpha /(1-q)}$ for all $\lambda>\lambda_{0}$. Therefore, from (3.8) we get $L u_{\lambda} \leq \lambda a u_{\lambda}^{p}-b u_{\lambda}^{q}$ in $\Omega \times \mathbb{R}$.

On the other hand, since we can construct supersolutions $w_{\lambda}$ of (1.3) such that $w_{\lambda} \geq c$ in $\Omega \times \mathbb{R}$ for all $c>0$ and all $\lambda>0$ (see [12, Lemma 2.3]), Theorem 1 in [7] applies and thus the first assertion of the theorem is proved.

Let $\lambda>\lambda_{0}$, let $u_{\lambda}$ be the solution of (1.3) found above, and let $m_{\lambda}:=\lambda p a u_{\lambda}^{p-1}-q b u_{\lambda}^{q-1}, \widetilde{m}_{\lambda}:=\lambda a u_{\lambda}^{p-1}-b u_{\lambda}^{q-1}$. Note that (H2) and (H3) imply that $m_{\lambda}$ and $\widetilde{m}_{\lambda}$ belong to $L_{T}^{s}$ for some $s>N+2$. We claim that the implicit function theorem can be applied in the point $\left(\lambda, u_{\lambda}\right)$. Indeed, to see this it suffices to show that for a given $g \in L_{T}^{r}$ there is a unique solution $h \in W_{r, T}^{2,1}$ of the problem

$$
\begin{cases}\left(L-m_{\lambda}\right) h=g & \text { in } \Omega \times \mathbb{R} \\ h=0 & \text { on } \partial \Omega \times \mathbb{R} \\ h T \text {-periodic, } & \end{cases}
$$

and that the solution operator $S_{\lambda}$ for this problem is continuous. Consider first the case when $\lambda_{1}\left(m_{\lambda}\right)$ exists. From (1.3) it follows that $\lambda_{1}\left(\widetilde{m}_{\lambda}\right)=1$. Since $\widetilde{m}_{\lambda} \geq m_{\lambda} / q$, recalling the comparison principle in Remark 2.2 (iv) we obtain

$$
1 \leq q \lambda_{1}\left(m_{\lambda}\right)<\lambda_{1}\left(m_{\lambda}\right)
$$

and so $\mu_{m_{\lambda}}(1)>0$. If $\lambda_{1}\left(m_{\lambda}\right)$ does not exist the same conclusion holds. Hence, by the results in Remark 2.2 (iii) and Remark 2.1 (ii) in both cases $S_{\lambda}$ is well defined and continuous on $W_{r, T}^{2,1}$.

Let $(\alpha, \beta)$ be a maximal interval in which $\lambda \rightarrow u_{\lambda}$ is a $C^{1}$ map into $W_{r, T}^{2,1} \cap P^{\circ}$. Observe that $\lambda \rightarrow u_{\lambda}$ is increasing. Indeed, differentiating (1.3) with respect to $\lambda$ gives

$$
\left(L-m_{\lambda}\right) \frac{\partial u}{\partial \lambda}=b u_{\lambda}^{p} \geq 0 \quad \text { in } \Omega \times \mathbb{R} .
$$

If $\lambda_{1}\left(m_{\lambda}\right)$ exists, Remark 2.2 (iii) and (3.10) imply $\frac{\partial u}{\partial \lambda}>0$. If $\lambda_{1}\left(m_{\lambda}\right)$ does not exist, then $\mu_{m_{\lambda}}$ is positive everywhere, thus $\mu_{m_{\lambda}}(1)>0$ and so, again by Remark 2.2 (iii) we get $\frac{\partial u}{\partial \lambda}>0$ also in this case.

Now, suppose $\beta<\infty$, and let $u_{j} \in P^{\circ}$ be the solutions of (1.3) corresponding to some sequence $\lambda_{j} \rightarrow \beta^{-}$. Recalling Remark 2.1 (i), a standard compactness argument gives some $u_{\beta}$ solution of (1.3) for $\lambda=\beta$. 
Moreover, since $\lambda \rightarrow u_{\lambda}$ is increasing we have $u_{\beta} \in P^{\circ}$. But then reasoning as above we can apply the implicit function theorem in the point $\left(\beta, u_{\beta}\right)$, contradicting the maximality of $(\alpha, \beta)$.

Concerning the matter of uniqueness or multiplicity of positive solutions, there are examples of different nonnegative solutions for similar problems to (1.3) with $p=q$ both in the elliptic and the periodic parabolic case (cf. [2], [16]), so we cannot expect uniqueness here. Moreover, when $p>q, a=b \equiv 1, L=-\Delta_{p}$ and $N=1$, it is shown in [8] that for some $\lambda$ there are exactly two solutions in the interior of the positive cone for (1.3), and therefore we cannot expect a general uniqueness theorem for the case $p>q$ neither. However, when $p \leq q$ the solution in the interior of the positive cone for (1.3) (if such a solution exists) turns out to be unique as the following theorem shows. Its proof is inspired in the proof of Proposition 2.2 in [6] and uses a change of variable introduced (for the elliptic case and $b=0$ ) by L. Nirenberg (see [3, Appendix II, Method IV]).

Theorem 3.5. Let $0<p \leq q<1, \lambda>0$, and let $0 \leq a, b \in L_{T}^{r}$, $r>N+2$. Then there exists at most one solution $u \in W_{r, T}^{2,1} \cap P^{\circ}$ of $(1.3)$.

Proof: Suppose that there exist $u, v \in W_{r, T}^{2,1} \cap P^{\circ}$ solutions of (1.3), and let $C:=\{(x, t) \in \Omega \times \mathbb{R}: u(x, t)>v(x, t)\}$. Let $s$ be such that $p \leq s \leq q$. A simple computation gives that $h(\xi):=\left(\lambda a(x, t) \xi^{p}-b(x, t) \xi^{q}\right) / \xi^{s}$ is nonincreasing in $(0, \infty)$ a.e. $(x, t) \in \Omega \times \mathbb{R}$. For $(x, t) \in C$, define $w(x, t):=\int_{v(x, t)}^{u(x, t)} \xi^{-s} d \xi$. Then clearly $w=0$ on $\partial C$ and taking into account that $u, v \in P^{\circ}$ it also follows that $w$ is continuous in $\bar{C}$. Now, since $h(\xi)$ and $\xi^{s-1}$ are nonincreasing, a computation shows that, in $C$,

$$
\begin{aligned}
L w-c_{0} w= & u^{-s} L u-v^{-s} L v+s u^{-s-1}\langle A \nabla u, \nabla u\rangle \\
& -s v^{-s-1}\langle A \nabla v, \nabla v\rangle-c_{0}\left(u^{1-s}-v^{1-s}\right) \\
\leq & h(u)-h(v)+s u^{-s-1}\langle A \nabla u, \nabla u\rangle-s u^{s-1} v^{-2 s}\langle A \nabla v, \nabla v\rangle \\
\leq & s u^{s-1}\left\langle\nabla u / u^{s}+\nabla v / v^{s}, A \nabla w\right\rangle .
\end{aligned}
$$

Thus, recalling the maximum principle in Lemma 2.3 and Remark 2.4, the theorem follows.

\section{References}

[1] S. Alama and G. Tarantello, Elliptic problems with nonlinearities indefinite in sign, J. Funct. Anal. 141(1) (1996), 159-215. 
[2] C. Bandle, M. A. Pozio And A. Tesei, The asymptotic behavior of the solutions of degenerate parabolic equations, Trans. Amer. Math. Soc. 303(2) (1987), 487-501.

[3] H. Brezis and S. Kamin, Sublinear elliptic equations in $\mathbb{R}^{n}$, Manuscripta Math. 74(1) (1992), 87-106.

[4] D. DANERs, Domain perturbation for linear and nonlinear parabolic equations, J. Differential Equations 129(2) (1996), 358-402.

[5] D. Daners And P. Koch Medina, "Abstract evolution equations, periodic problems and applications", Pitman Research Notes in Mathematics Series 279, Longman Scientific \& Technical, Harlow; copublished in the United States with John Wiley \& Sons, Inc., New York, 1992.

[6] M. Delgado and A. Suárez, On the uniqueness of positive solution of an elliptic equation, Appl. Math. Lett. 18(10) (2005), 1089-1093.

[7] J. Deuel and P. Hess, Nonlinear parabolic boundary value problems with upper and lower solutions, Israel J. Math. 9(1) (1978), $92-104$

[8] J. I. DÍAz AND J. HeRnÁndez, Global bifurcation and continua of nonnegative solutions for a quasilinear elliptic problem, C. R. Acad. Sci. Paris Sér. I Math. 329(7) (1999), 587-592.

[9] D. Gilbarg and N. S. Trudinger, "Elliptic partial differential equations of second order", Second edition, Grundlehren der Mathematischen Wissenschaften 224, Springer-Verlag, Berlin, 1983.

[10] T. Godoy and U. Kaufmann, On principal eigenvalues for periodic parabolic problems with optimal condition on the weight function, J. Math. Anal. Appl. 262(1) (2001), 208-220.

[11] T. Godoy and U. Kaufmann, On positive solutions for some semilinear periodic parabolic eigenvalue problems, J. Math. Anal. Appl. 277(1) (2003), 164-179.

[12] T. Godoy and U. Kaufmann, On the existence of positive solutions for periodic parabolic sublinear problems, Abstr. Appl. Anal. 17 (2003), 975-984.

[13] T. Godoy, U. Kaufmann and S. Paczka, Positive solutions for sublinear periodic parabolic problems, Nonlinear Anal. 55(1-2) (2003), 73-82.

[14] J. Hernández, F. Mancebo and J. M. Vega, Positive solutions for singular nonlinear elliptic equations, Proc. Roy. Soc. Edinburgh Sect. A (to appear).

[15] P. Hess, "Periodic-parabolic boundary value problems and positivity", Pitman Research Notes in Mathematics Series 247, Longman 
Scientific \& Technical, Harlow; copublished in the United States with John Wiley \& Sons, Inc., New York, 1991.

[16] P. Hess, M. A. Pozio And A. Tesei, Time periodic solutions for a class of degenerate parabolic problems, Houston J. Math. 21(2) (1995), 367-394.

[17] N. Hirano and W. S. Kim, Multiple existence of periodic solutions for a nonlinear parabolic problem with singular nonlinearities, Nonlinear Anal. 54(3) (2003), 445-456.

[18] O. A. Ladyženskaja, V. A. Solonnikov and N. N. URaL'CEVA, "Linear and quasilinear equations of parabolic type", (Russian), Translated from the Russian by S. Smith, Translations of Mathematical Monographs 23, American Mathematical Society, Providence, R.I., 1967.

[19] G. M. Lieberman, Time-periodic solutions of linear parabolic differential equations, Comm. Partial Differential Equations 24(3-4) (1999), 631-663.

[20] L. Moschini, S. I. Pohozaev And A. Tesei, Existence and nonexistence of solutions of nonlinear Dirichlet problems with first order terms, J. Funct. Anal. 177(2) (2000), 365-382.

Facultad de Matemática, Astronomía y Física

Universidad Nacional de Córdoba

Ciudad Universitaria

5000 Córdoba

Argentina

E-mail address: godoy@mate.uncor.edu

E-mail address: kaufmann@mate.uncor.edu 